Optimal critical mass for the two-dimensional keller-segel model with rotational flux terms

ELIO ESPEJO AND HAO WU

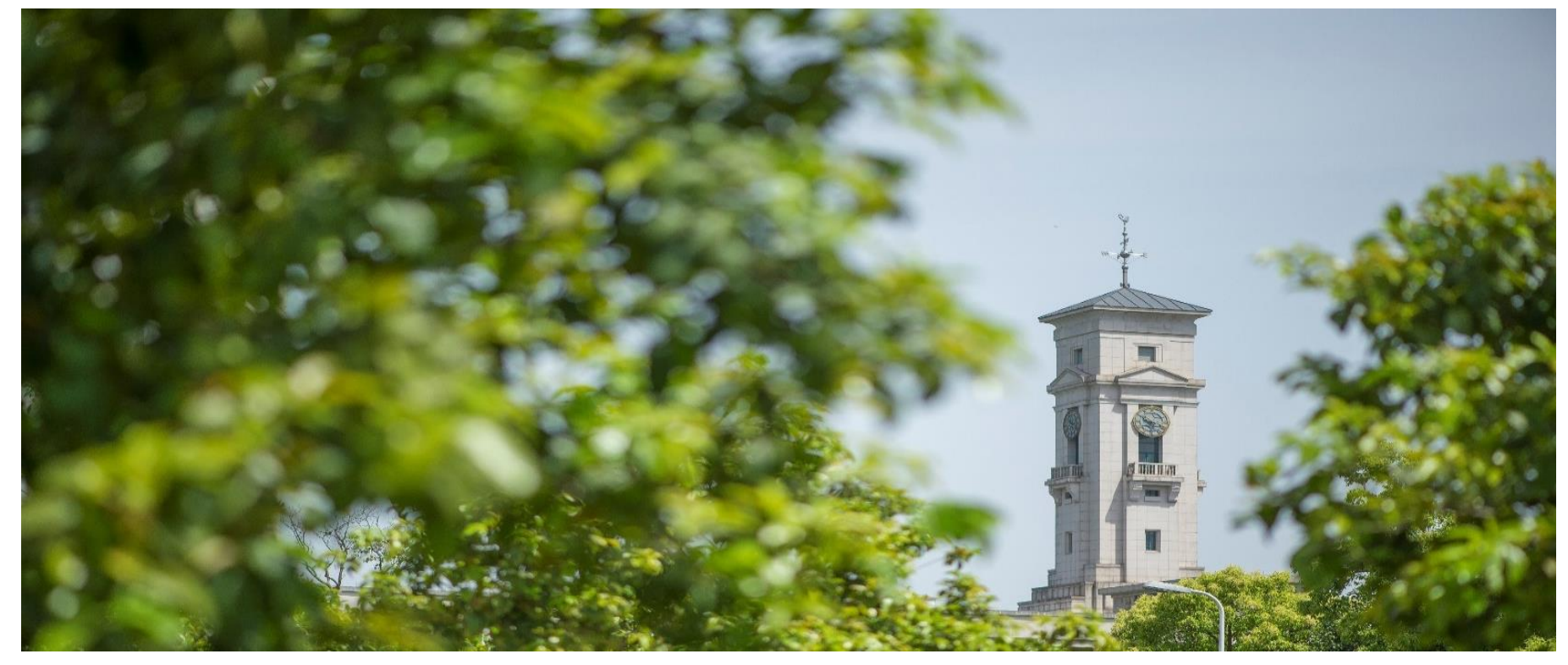


University of Nottingham Ningbo China, 199 Taikang East Road, Ningbo, 315100, China

First published 2020

This work is made available under the terms of the Creative Commons Attribution 4.0 International License:

http://creativecommons.org/licenses/by/4.0

The work is licenced to the University of Nottingham Ningbo China under the Global University Publication Licence:

https://www.nottingham.edu.cn/en/library/documents/researchsupport/global-university-publications-licence.pdf Nottingham 


\title{
OPTIMAL CRITICAL MASS FOR THE TWO-DIMENSIONAL KELLER-SEGEL MODEL WITH ROTATIONAL FLUX TERMS*
}

\author{
ELIO ESPEJO ${ }^{\dagger}$ AND HAO WU
}

\begin{abstract}
Our aim is to show that several important systems of partial differential equations arising in mathematical biology, fluid dynamics and electrokinetics can be approached within a single model, namely, a Keller-Segel-type system with rotational flux terms. In particular, we establish sharp conditions on the optimal critical mass for having global existence and finite time blow-up of solutions in two spatial dimensions. Our results imply that the rotated chemotactic response can delay or even avoid the blow-up. The key observation is that for any angle of rotation $\alpha \in(-\pi, \pi]$, the resulting PDE system preserves a dissipative energy structure. Inspired by this property, we also provide an alternative derivation of the general system via an energetic variational approach.
\end{abstract}

Keywords. chemotaxis; rotational flux; critical mass; blow-up; global existence; dissipative energy structure.

AMS subject classifications. 35K57; 35B40; 92C15; 92C17.

\section{Introduction}

We consider the Cauchy problem of the following generalized Keller-Segel system in $\mathbb{R}^{2}$ :

$$
\begin{aligned}
\rho_{t} & =\Delta \rho-\chi \operatorname{div}(\rho A \nabla c), & & (x, t) \in \mathbb{R}^{2} \times \mathbb{R}^{+}, \\
-\Delta c & =\rho, & & (x, t) \in \mathbb{R}^{2} \times \mathbb{R}^{+}, \\
\rho(x, 0) & =\rho_{0}(x), & & x \in \mathbb{R}^{2},
\end{aligned}
$$

where $\chi$ is a positive constant and $A$ denotes a $2 \times 2$ matrix given by

$$
A:=\left(\begin{array}{cc}
\cos \alpha & -\sin \alpha \\
\sin \alpha & \cos \alpha
\end{array}\right) \text { with } \alpha \in(-\pi, \pi] \text { being a constant. }
$$

The system (1.1)-(1.2) generalizes the well-known Keller-Segel (or Patlak-Keller-Segel) model that describes the oriented movement of bacterial cells in response to certain chemical signals $[20,26]$. When the angle of rotation $\alpha=0$, the matrix $A$ simply reduces to the $2 \times 2$ identity matrix $I$, and as a consequence, the standard parabolic-elliptic Patlak-Keller-Segel system is recovered (see, e.g., [3,4]). In the context of mathematical biology, the unknown variable $\rho$ in system (1.1)-(1.2) denotes the density of bacteria and $c$ represents the concentration of chemoattractant. Equation (1.1) indicates that the motion of bacteria is driven by the self-diffusion and the gradient of concentration of the chemoattractant, while the Poisson Equation (1.2) means that the chemoattractant is produced by the cells themselves and it is diffusing into the environment. We remark

†School of Mathematical Sciences, The University of Nottingham Ningbo China, Taikang East Road 199, Ningbo 315100, China (elio-eduardo-espejo.arenas@nottingham.edu.cn).

$\ddagger$ School of Mathematical Sciences and Shanghai Key Laboratory for Contemporary Applied Mathematics, Fudan University, Handan Road 220, Shanghai 200433, China;(haowufd@fudan.edu.cn). 
that a possible derivative of $c$ with respect to time is neglected from the left-hand side of (1.2), as one usually makes the assumption that the process of chemical diffusion is much faster than the time scale of cell movement (see e.g., [19]). There is a vast literature about the Keller-Segel model and its variants, for more detailed information, we refer the reader to survey papers $[1,15-17]$ and the references therein.

One feature of our system (1.1)-(1.2) is that the chemotactic sensitivity turns out to be a tensor $\chi A$ instead of a scalar function like in the classical Keller-Segel model. This tensor-valued sensitivity is motivated by some interesting biased chemotactic response that has been observed for different kinds of bacteria in experiments, see for instance, $[9,12]$, such that when the bacteria swim close to a surface, they may be subject to a net rotational force and form spiral-type patterns. In this situation, the effect of chemotaxis is not precisely oriented along the concentration gradient (i.e., $\nabla c$ ), but involves certain rotational flux component. Taking this fact into account, the following partial differential equation was derived in [34] from a microscopic model using asymptotic analysis (see [34, (5.26)] for a slightly more general form):

$$
\rho_{t}=\Delta \rho-\operatorname{div}\left[\rho\left(\chi_{1} \nabla c+\chi_{2} \nabla^{\perp} c\right)\right]
$$

where $\chi_{1}, \chi_{2}$ are constants that are not zero at the same time. The anti-gradient term given by

$$
\nabla^{\perp} c:=\left(\frac{\partial c}{\partial x_{2}},-\frac{\partial c}{\partial x_{1}}\right)^{T}
$$

stands for the possible swimming bias of bacteria with respect to the gradient of chemical concentration. It is easy to see that Equation (1.5) can be written as

$$
\rho_{t}=\Delta \rho-\chi \operatorname{div}(\rho A \nabla c)
$$

where $\chi:=\sqrt{\chi_{1}^{2}+\chi_{2}^{2}}>0$ and $A$ is an orthogonal matrix such that

$$
A=\left(\begin{array}{ll}
\chi_{1} / \chi & \chi_{2} / \chi \\
-\chi_{2} / \chi & \chi_{1} / \chi
\end{array}\right)
$$

Set

$$
\alpha= \begin{cases}\arcsin \left(\frac{\chi_{2}}{\chi}\right)+\pi, & \text { if } \frac{\chi_{1}}{\chi}<0, \frac{\chi_{2}}{\chi} \leq 0, \\ -\arcsin \left(\frac{\chi_{2}}{\chi}\right), & \text { if } \frac{\chi_{1}}{\chi} \geq 0, \\ \arcsin \left(\frac{\chi_{2}}{\chi}\right)-\pi, & \text { if } \frac{\chi_{1}}{\chi}<0, \frac{\chi_{2}}{\chi}>0 .\end{cases}
$$

Then Equation (1.5) simply reduces to (1.1). We see that the positive constant $\chi$ measures the strength of chemotactic sensitivity, while the matrix $A$ characterizes the rotational effect with respect to the concentration gradient of the chemoattractant with $\alpha \in(-\pi, \pi]$ being the angle of rotation.

Interestingly, by taking different values of $\alpha$ in system (1.1)-(1.2), we are able to recover several important systems of partial differential equations in the literature. First, as we have mentioned above, the standard parabolic-elliptic Patlak-Keller-Segel system can be obtained with $\alpha=0$. Next, for the choice of $\alpha=\pi$, system (1.1)-(1.2) yields a Keller-Segel model involving certain repelling chemotaxis. On the other hand, we note that in a different physical context, the same system corresponds to the classical 
drift-diffusion model with Poisson-coupling for an electron gas (neglecting the confining potential), see e.g., [22]. Finally, let us recall the Navier-Stokes system for an incompressible Newtonian fluid

$$
\begin{aligned}
u_{t}+u \cdot \nabla u-\Delta u+\nabla p & =0, \\
\operatorname{div} u & =0,
\end{aligned}
$$

in $\mathbb{R}^{3} \times \mathbb{R}^{+}$, where $u=\left(u_{1}, u_{2}, u_{3}\right)^{T}$ is the fluid velocity and $p$ denotes the pressure. A classical procedure to analyze the two-dimensional dynamics of the fluid is done by taking $x=\left(x_{1}, x_{2}, 0\right)^{T}$ and $u_{3}=0$. In this case, the vorticity is given by $\nabla \times u=(0,0, \omega)^{T}$ with $\omega=\frac{\partial u_{2}}{\partial x_{1}}-\frac{\partial u_{1}}{\partial x_{2}}$. Applying the curl operator to Equations (1.7)-(1.8) and using the Biot-Savart law, we then (formally) obtain the vorticity equation for a two-dimensional flow (see e.g., [13, Sections 2.1.2, 2.1.3])

$$
\omega_{t}=\operatorname{div}\left[\nabla \omega-\omega\left(\nabla^{\perp} K * \omega\right)\right]
$$

in $\mathbb{R}^{2} \times \mathbb{R}^{+}$, where

$$
K(x):=-\frac{1}{2 \pi} \log |x|, \quad x=\left(x_{1}, x_{2}\right)^{T} \in \mathbb{R}^{2}, \quad x \neq 0,
$$

is the two-dimensional Newtonian potential. In particular, the above equation for $\omega$ can be alternatively written in the following form

$$
\begin{aligned}
\omega_{t} & =\Delta \omega-\operatorname{div}(\omega A \nabla v), \\
-\Delta v & =\omega,
\end{aligned}
$$

with the matrix

$$
A:=\left(\begin{array}{ll}
0 & 1 \\
-1 & 0
\end{array}\right)
$$

Hence, we easily see that the two-dimensional scalar vorticity equation can be deduced by taking $\chi=1$ and the rotation angle $\alpha=-\pi / 2$ in our system (1.1)-(1.2).

Throughout this paper, the chemotactic sensitivity in system (1.1)-(1.2) is assumed to be a matrix with constant components for the sake of simplicity. For the special case $A=I$ (which actually yields a scalar chemotactic sensitivity function), extensive studies have been made in the literature, we refer to $[3,4,8,11,23-25]$ and the references cited therein. In general, according to the respective modeling background, the chemotactic sensitivity function may depend on $\rho, c$ and also on the spatial and temporal variables $x$, $t$. Many results describing sufficient conditions for the existence of global solutions for chemotaxis models with general tensor-valued sensitivities/rotational flux terms have been obtained recently, see for instance, $[5,10,21,28,32,35]$ and their references. We also refer to $[6,29-31,33]$ for related results on generalized chemotaxis systems involving fluid interactions.

Nevertheless, to the best of our knowledge, there is no blow-up result for the KellerSegel-type model (1.1)-(1.2) in the literature if $A$ does not represent the identity matrix I. Our aim is to approach this problem when $A$ takes the form of a rotational matrix with constant components (see (1.4)). As we have seen above, this simple setting already includes several PDE systems of great interest. Besides, here we choose to consider the Cauchy problem in the whole space $\mathbb{R}^{2}$, since we wish to focus on the possible influences due to rotation but are not interested in boundary effects. 
When $A=I$ (i.e., $\alpha=0$ ), it has been shown in [4] that the value $\frac{8 \pi}{\chi}$ serves as an optimal critical mass for system (1.1)-(1.2): for any nonnegative initial datum $\rho_{0}$ of finite second moment and entropy, the condition $\int_{\mathbb{R}^{2}} \rho_{0}(x) \mathrm{d} x<\frac{8 \pi}{\chi}$ implies the existence of global (free-energy) solutions, while in the case $\int_{\mathbb{R}^{2}} \rho_{0}(x) \mathrm{d} x>\frac{8 \pi}{\chi}$ the solutions may blow-up in finite time. It is clear that the critical mass measures the balance between the tendency of bacteria to spread in $\mathbb{R}^{2}$ due to diffusion and the tendency of bacteria to aggregate because of the drift induced by the chemoattractivity. Then concerning the case for a general rotational matrix $A$ as in (1.4), a natural question arises: Can a rotated chemotactic response delay or even avoid the blow-up? We will give a positive answer to this question. More precisely, for the initial value problem (1.1)-(1.3), we obtain sufficient conditions for having global free-energy solutions and find the optimal critical mass that can be precisely determined by the rotation angle $\alpha$, leading to possible finite time blow-up.

The main result of this paper is as follows:

TheOrem 1.1 (Optimal critical mass, finite time blow-up and global existence). Assume that the initial datum $\rho_{0}$ satisfies

$$
0 \leq \rho_{0} \in L^{1}\left(\mathbb{R}^{2},\left(1+|x|^{2}\right) \mathrm{d} x\right), \quad \rho_{0} \log \rho_{0} \in L^{1}\left(\mathbb{R}^{2}, \mathrm{~d} x\right),
$$

$\chi>0$ and $A$ is a $2 \times 2$ matrix given by (1.4). For every rotation angle $\alpha \in(-\pi, \pi]$, the Cauchy problem (1.1)-(1.3) admits a unique a free-energy solution $(\rho, c)$ (see Definition 3.1 below) on the maximal time interval $\left[0, T_{\max }\right)$. Moreover, denoting the initial total mass by

$$
M:=\int_{\mathbb{R}^{2}} \rho_{0}(x) \mathrm{d} x
$$

we have the following conclusions:

(1) If $\alpha \in\left(-\frac{\pi}{2}, \frac{\pi}{2}\right)$ and $M>\frac{8 \pi}{\chi \cos \alpha}$, then

$$
0<T_{\max } \leq \frac{2 \pi}{[\chi(\cos \alpha) M-8 \pi] M} \int_{\mathbb{R}^{2}} \rho_{0}(x)|x|^{2} \mathrm{~d} x<+\infty
$$

and

$$
\lim _{t \nearrow T_{\max }} \int_{\mathbb{R}^{2}} \rho(x, t) \log \rho(x, t) \mathrm{d} x=+\infty
$$

(2) If $\alpha \in\left(-\frac{\pi}{2}, \frac{\pi}{2}\right)$ and $0 \leq M<\frac{8 \pi}{\chi \cos \alpha}$, then $T_{\max }=+\infty$.

(3) If $\alpha \in\left(-\pi,-\frac{\pi}{2}\right] \cup\left[\frac{\pi}{2}, \pi\right]$, then for all $M \geq 0$, it holds $T_{\max }=+\infty$.

Theorem 1.1 implies that the rotational effect (characterized by the rotation angle $\alpha$ ) plays an essential role in the blow-up/global existence for problem (1.1)-(1.3) with integrable initial data of finite second moment and entropy. In particular, for $\alpha \in$ $\left(-\frac{\pi}{2}, \frac{\pi}{2}\right)$, we obtain a sharp result describing the possibility of having global existence versus having a blow-up in finite time, with the optimal critical mass given by $\frac{8 \pi}{\chi \cos \alpha}$. While for $\alpha \in\left(-\pi,-\frac{\pi}{2}\right] \cup\left[\frac{\pi}{2}, \pi\right]$, we show that the free-energy solutions to problem (1.1)(1.3) always exist globally in time. The key to the proof relies on the observation that under rotational effects the chemotaxis system (1.1)-(1.2) still enjoys a dissipative energy structure for all $\alpha \in(-\pi, \pi]$ (see Lemma 2.1 below), such that the associated free 
energy given by

$$
E_{\alpha}(\rho, c):=\int_{\mathbb{R}^{2}} \rho \log \rho \mathrm{d} x-\frac{\chi \cos \alpha}{2} \int_{\mathbb{R}^{2}} \rho c \mathrm{~d} x
$$

is decreasing in time. This crucial property enables us to work within the framework of free-energy solutions (see Definition 3.1) and extend previous works on the classical parabolic-elliptic Keller-Segel system with $\alpha=0$ (e.g., [4,11]) to an arbitrary rotation angle. We remark that the case subject to the critical mass is more involved. For problem (1.1)-(1.3) with $\alpha=0$, in [3], the authors proved global existence of free-energy solutions with the critical mass $\frac{8 \pi}{\chi}$ and moreover, the infinite time aggregation as $t \rightarrow$ $+\infty$. We expect that similar results still hold for the general case $\alpha \in\left(-\frac{\pi}{2}, \frac{\pi}{2}\right)$ with the corresponding critical mass $\frac{8 \pi}{\chi \cos \alpha}$. Besides, the dissipative energy structure also motives us to expect that the large-time behavior, e.g., convergence of global solutions to asymptotically self-similar profiles in rescaled variables that has been observed for the Keller-Segel system [4], the drift-diffusion-Poisson system [2] and the vorticity equation [13], can be extended to the general case $\alpha \in(-\pi, \pi]$. These issues will be illustrated in our future study.

Finally, it is worth mentioning that inspired by the dissipative energy structure, we are able to give an alternative derivation of system (1.1)-(1.2) by applying the energetic variational approach that combines the least action principle and Onsager's principle of maximum energy dissipation in continuum mechanics (see e.g., $[14,18]$ and references therein). In this way, we generalize the previous work [8] and show that system (1.1)-(1.2) can be viewed as a general diffusion model with nonlocal interactions and rotational effects. In particular, it naturally keeps important physical laws, such as mass conservation, energy dissipation and force balance.

The remaining part of this paper is organized as follows. In Section 2, we present the dissipative energy structure of system (1.1)-(1.2) and in Section 3 we give the proof of our main result Theorem 1.1. In the Appendix, we first sketch the proof of Proposition 3.1 on the existence and uniqueness of local free-energy solutions to the Cauchy problem (1.1)-(1.3) and then provide a brief derivation of system (1.1)-(1.2) via the energetic variational approach.

\section{Dissipative energy structure}

In this section, we show that for any rotation angle $\alpha \in(-\pi, \pi]$, the free energy $E_{\alpha}(\rho, c)$ enjoys a basic energy law such that it is monotone non-increasing with respect to time. More precisely, for sufficiently smooth solutions to problem (1.1)-(1.3) with fast decay at infinity, we have

LEMma 2.1 (Basic energy law). Let $(\rho, c)$ be a smooth solution to the Cauchy problem (1.1)-(1.3) with (1.4) such that $0 \leq \rho \in C\left([0, T) ; L^{1}\left(\mathbb{R}^{2}\right)\right), \rho\left(1+|x|^{2}\right), \rho \log \rho$ are bounded in $L^{\infty}\left(0, T ; L^{1}\left(\mathbb{R}^{2}\right)\right), \nabla \sqrt{\rho} \in L^{1}\left(0, T ; L^{2}\left(\mathbb{R}^{2}\right)\right)$ and $\nabla c \in L^{\infty}\left((0, T) \times \mathbb{R}^{2}\right)$. Then it holds

$$
\frac{\mathrm{d}}{\mathrm{d} t} E_{\alpha}(\rho, c)=-D_{\alpha}(\rho, c) \leq 0, \quad \forall t \in(0, T),
$$

where $E_{\alpha}(\rho, c)$ is defined by (1.13) and

$$
D_{\alpha}(\rho, c):=\int_{\mathbb{R}^{2}} \rho|\nabla \log \rho-\chi \cos \alpha \nabla c|^{2} \mathrm{~d} x .
$$


Proof. Integrating over $\mathbb{R}^{2}$ the Equation (1.1) for $\rho$, we get

$$
\frac{\mathrm{d}}{\mathrm{d} t} \int_{\mathbb{R}^{2}} \rho \mathrm{d} x=0, \quad \forall t \in(0, T) .
$$

This implies the mass conservation property of problem (1.1)-(1.3), i.e.,

$$
\int_{\mathbb{R}^{2}} \rho(x, t) \mathrm{d} x=\int_{\mathbb{R}^{2}} \rho(x, 0) \mathrm{d} x, \quad \forall t \in(0, T) .
$$

Next, we decompose the matrix $A$ given by (1.4) into the following form

$$
A=\cos \alpha I+\sin \alpha R,
$$

where

$$
I:=\left(\begin{array}{ll}
1 & 0 \\
0 & 1
\end{array}\right) \quad \text { and } \quad R:=\left(\begin{array}{ll}
0 & -1 \\
1 & 0
\end{array}\right) .
$$

Thus, it follows that

$$
\begin{aligned}
A \nabla c & =\cos \alpha \nabla c+\sin \alpha R \nabla c \\
& =\cos \alpha \nabla c-\sin \alpha \nabla^{\perp} c
\end{aligned}
$$

and Equation (1.1) can be written in the following form

$$
\begin{aligned}
\rho_{t} & =\Delta \rho-\chi \cos \alpha \operatorname{div}(\rho \nabla c)+\chi \sin \alpha \operatorname{div}\left(\rho \nabla^{\perp} c\right) \\
& =\operatorname{div}[\rho \nabla(\log \rho-\chi(\cos \alpha) c)]+\chi \sin \alpha \operatorname{div}\left(\rho \nabla^{\perp} c\right) .
\end{aligned}
$$

Multiplying (2.7) by $\log \rho-\chi(\cos \alpha) c$ and integrating over $\mathbb{R}^{2}$, we obtain

$$
\begin{aligned}
& \int_{\mathbb{R}^{2}} \rho_{t}[\log \rho-\chi(\cos \alpha) c] \mathrm{d} x \\
= & -\int_{\mathbb{R}^{2}} \rho|\nabla \log \rho-\chi(\cos \alpha) \nabla c|^{2} \mathrm{~d} x \\
& \quad-\chi \sin \alpha \int_{\mathbb{R}^{2}} \nabla[\log \rho-\chi(\cos \alpha) c] \cdot\left(\rho \nabla^{\perp} c\right) \mathrm{d} x .
\end{aligned}
$$

The second integral on the right-hand side of (2.8) simply vanishes since

$$
\begin{aligned}
& \int_{\mathbb{R}^{2}} \nabla[\log \rho-\chi(\cos \alpha) c] \cdot\left(\rho \nabla^{\perp} c\right) \mathrm{d} x \\
= & \int_{\mathbb{R}^{2}}\left[\nabla \rho \cdot \nabla^{\perp} c-\chi(\cos \alpha) \rho \nabla c \cdot \nabla^{\perp} c\right] \mathrm{d} x \\
= & \int_{\mathbb{R}^{2}}\left[-\rho \operatorname{div}\left(\nabla^{\perp} c\right)-\chi(\cos \alpha) \rho \nabla c \cdot \nabla^{\perp} c\right] \mathrm{d} x \\
= & 0 .
\end{aligned}
$$

On the other hand, since $c$ is the solution to the Poisson Equation (1.2), uniquely determined up to a harmonic function, we can just take

$$
c(x, t)=\rho * K=\int_{\mathbb{R}^{2}} K(x-y) \rho(y, t) \mathrm{d} y,
$$


with the Green kernel function $K$ given by (1.9). Then from (1.13), (2.3) and (2.10) we see that the left-hand side of (2.8) can be written as

$$
\begin{aligned}
& \int_{\mathbb{R}^{2}} \rho_{t}[\log \rho-\chi(\cos \alpha) c] \mathrm{d} x \\
= & \int_{\mathbb{R}^{2}}\left(\rho_{t} \log \rho+\rho_{t}\right) \mathrm{d} x-\chi \cos \alpha \int_{\mathbb{R}^{2}} \int_{\mathbb{R}^{2}} \rho_{t}(x, t) \rho(y, t) K(x-y) \mathrm{d} x \mathrm{~d} y \\
= & \frac{\mathrm{d}}{\mathrm{d} t}\left(\int_{\mathbb{R}^{2}} \rho \log \rho \mathrm{d} x-\frac{\chi \cos \alpha}{2} \int_{\mathbb{R}^{2}} \int_{\mathbb{R}^{2}} \rho(x, t) \rho(y, t) K(x-y) \mathrm{d} x \mathrm{~d} y\right) \\
= & \frac{\mathrm{d}}{\mathrm{d} t} E_{\alpha}(\rho, c) .
\end{aligned}
$$

Hence, we infer from (2.8) and (2.11) that

$$
\frac{\mathrm{d}}{\mathrm{d} t} E_{\alpha}(\rho, c)=-\int_{\mathbb{R}^{2}} \rho|\nabla \log \rho-\chi \cos \alpha \nabla c|^{2} \mathrm{~d} x, \quad \forall t \in(0, T),
$$

which immediately yields our conclusion (2.1)-(2.2).

\section{Proof of Theorem 1.1}

The existence of free-energy $E_{\alpha}(\rho, c)$ for system (1.1)-(1.2) motivates us to extend the approach of free-energy solutions for Keller-Segel models in the whole space $\mathbb{R}^{2}$, see for instance, $[3,4,11]$.

As in the proof of Lemma 2.1, the solution $c$ to the Poisson Equation (1.2) is defined directly by (recall (1.9), (2.10))

$$
c(x, t)=\rho * K=-\frac{1}{2 \pi} \int_{\mathbb{R}^{2}} \log |x-y| \rho(y, t) \mathrm{d} y .
$$

Then system (1.1)-(1.2) can be viewed as a single parabolic equation for $\rho$ with a nonlocal interaction term:

$$
\rho_{t}=\Delta \rho-\chi \operatorname{div}[\rho A(\rho * \nabla K)]
$$

Correspondingly, the free energy $E_{\alpha}(\rho, c)$ (see (1.13)) and the energy dissipation $D_{\alpha}(\rho, c)$ (see $(2.2)$ ) can be reduced to

$$
\begin{aligned}
\mathcal{E}_{\alpha}(\rho) & :=\int_{\mathbb{R}^{2}} \rho \log \rho \mathrm{d} x+\frac{\chi \cos \alpha}{4 \pi} \int_{\mathbb{R}^{2}} \int_{\mathbb{R}^{2}} \rho(x, t) \rho(y, t) \log |x-y| \mathrm{d} x \mathrm{~d} y, \\
\mathcal{D}_{\alpha}(\rho) & :=\int_{\mathbb{R}^{2}} \rho|\nabla \log \rho-\chi \cos \alpha(\rho * \nabla K)|^{2} \mathrm{~d} x .
\end{aligned}
$$

Keeping these facts in mind, we proceed to introduce the following concept of freeenergy solution to problem (1.1)-(1.3) (cf. $[3,4,11])$.

Definition 3.1 (Free-energy solution). Assume that $\chi>0$ and $A$ is a matrix given by (1.4). For any $T>0$ and any initial datum $\rho_{0}$ satisfying (1.12), we say that the nonnegative function $\rho \in L^{\infty}\left(0, T ; L^{1}\left(\mathbb{R}^{2}\right)\right) \cap C\left([0, T) ; \mathcal{D}^{\prime}\left(\mathbb{R}^{2}\right)\right)$ is a free-energy solution to the initial value problem (1.1)-(1.3) on the interval $(0, T)$, whenever the function $\rho$ satisfies $\left(1+|x|^{2}+|\log \rho|\right) \rho \in L^{\infty}\left(0, T ; L^{1}\left(\mathbb{R}^{2}\right)\right)$, Equation (3.2) is satisfied in the distributional sense such that

$$
\int_{\mathbb{R}^{2}} \rho_{0}(x) \phi(x, 0) \mathrm{d} x=\int_{0}^{T} \int_{\mathbb{R}^{2}} \rho\left\{[\nabla \log \rho-\chi A(\rho * \nabla K)] \cdot \nabla \phi-\phi_{t}\right\} \mathrm{d} x \mathrm{~d} t
$$


for any test function $\phi \in C_{0}^{2}\left([0, T) \times \mathbb{R}^{2}\right)$, moreover, the following energy inequality holds

$$
\mathcal{E}_{\alpha}(\rho(t))+\int_{0}^{t} \mathcal{D}_{\alpha}(\rho(s)) \mathrm{d} s \leq \mathcal{E}_{\alpha}\left(\rho_{0}\right), \quad \text { for a.e. } t \in(0, T),
$$

where $\mathcal{E}_{\alpha}, \mathcal{D}_{\alpha}$ are defined by (3.3) and (3.4), respectively.

First, we state a result on the existence and uniqueness of local-in-time free-energy solutions to problem (1.1)-(1.3) for all $\alpha \in(-\pi, \pi]$, without any restriction on the initial mass $\int_{\mathbb{R}^{2}} \rho_{0} \mathrm{~d} x$, which also gives a characterization on the maximal time of existence via the entropy functional.

Proposition 3.1 (Existence and uniqueness of local free-energy solution). Assume that $\chi>0$ and $A$ is a matrix given by (1.4). For any initial datum $\rho_{0}$ that satisfies (1.12), there exists a maximal time of existence $T_{\max }>0$ such that on $\left[0, T_{\max }\right)$, problem $(1.1)-$ (1.3) admits a unique free-energy solution in the sense of Definition 3.1. Moreover, we have the following criterion of extensibility: if $T_{\max }<+\infty$, then

$$
\lim _{t \nearrow T_{\max }} \int_{\mathbb{R}^{2}} \rho(x, t) \log \rho(x, t) \mathrm{d} x=+\infty
$$

Remark 3.1. Proposition 3.1 extends the known results on existence and uniqueness of local free-energy solutions to problem (1.1)-(1.3) for the special case $\alpha=0$ that have been obtained respectively in $[3,4]$ and [11]. We postpone its proof to the Appendix.

Next, we provide an estimate on the second moment of $\rho$, which will be useful for the study of blow-up of problem (1.1)-(1.3).

LEMMA 3.1 (Moment estimate). Let $\rho$ be a free-energy solution to problem (1.1)-(1.3) with initial datum $\rho_{0}$ satisfying (1.12) defined on the time interval $\left[0, T_{\max }\right)$. Then it holds

$$
\int_{\mathbb{R}^{2}} \rho(x, t)|x|^{2} \mathrm{~d} x=\frac{\chi \cos \alpha}{2 \pi} M\left(\frac{8 \pi}{\chi \cos \alpha}-M\right) t+\int_{\mathbb{R}^{2}} \rho_{0}(x)|x|^{2} \mathrm{~d} x, \quad \forall t \in\left[0, T_{\max }\right),
$$

where $M:=\int_{\mathbb{R}^{2}} \rho_{0}(x) \mathrm{d} x$.

Proof. For simplicity, below we just perform formal calculations, which can be justified rigorously by applying the approximating argument as in [4, Lemma 2.1], using a suitable weak formulation of $\rho$ together with Lebesgue's dominated convergence theorem (see also [3, Lemma 2.4]).

Multiplying Equation (3.2) by $|x|^{2}$ and integrating over $\mathbb{R}^{2}$, we obtain

$$
\begin{aligned}
\frac{\mathrm{d}}{\mathrm{d} t} \int_{\mathbb{R}^{2}} \rho|x|^{2} \mathrm{~d} x & =\int_{\mathbb{R}^{2}}|x|^{2} \Delta \rho \mathrm{d} x-\chi \int_{\mathbb{R}^{2}}|x|^{2} \operatorname{div}[\rho A(\rho * \nabla K)] \mathrm{d} x \\
& =4 \int_{\mathbb{R}^{2}} \rho \mathrm{d} x+2 \chi \int_{\mathbb{R}^{2}} x \cdot[\rho A(\rho * \nabla K)] \mathrm{d} x \\
& =4 \int_{\mathbb{R}^{2}} \rho_{0} \mathrm{~d} x-2 \chi \int_{\mathbb{R}^{2}} x \cdot\left(\rho A \int_{\mathbb{R}^{2}} \frac{1}{2 \pi} \frac{x-y}{|x-y|^{2}} \rho(y, t) \mathrm{d} y\right) \mathrm{d} x \\
& =4 M-\frac{\chi}{\pi} \int_{\mathbb{R}^{2} \times \mathbb{R}^{2}} x \cdot\left(A \frac{x-y}{|x-y|^{2}}\right) \rho(x, t) \rho(y, t) \mathrm{d} y \mathrm{~d} x \\
& :=4 M-\frac{\chi}{\pi} J,
\end{aligned}
$$


where in the above identity we have also used the mass conservation property (2.3) and the definition of $M$. Interchanging $x$ and $y$ in the integral $J$, we get

$$
J=-\int_{\mathbb{R}^{2} \times \mathbb{R}^{2}} y \cdot\left(A \frac{x-y}{|x-y|^{2}}\right) \rho(x, t) \rho(y, t) \mathrm{d} y \mathrm{~d} x,
$$

which easily implies

$$
J=\frac{1}{2} \int_{\mathbb{R}^{2} \times \mathbb{R}^{2}}\left(\frac{x-y}{|x-y|}\right) \cdot A\left(\frac{x-y}{|x-y|}\right) \rho(x, t) \rho(y, t) \mathrm{d} y \mathrm{~d} x .
$$

On the other hand, noticing that for any $a, b \in \mathbb{R}$,

$$
\left(\begin{array}{ll}
a & b
\end{array}\right)\left(\begin{array}{cc}
\cos \alpha & -\sin \alpha \\
\sin \alpha & \cos \alpha
\end{array}\right)\left(\begin{array}{l}
a \\
b
\end{array}\right)=\left(a^{2}+b^{2}\right) \cos \alpha
$$

then we infer from (3.7) that

$$
J=\frac{\cos \alpha}{2} \int_{\mathbb{R}^{2} \times \mathbb{R}^{2}} \rho(x, t) \rho(y, t) \mathrm{d} y \mathrm{~d} x=\frac{\cos \alpha}{2} M^{2} .
$$

As a consequence, it follows from (3.6) and (3.8) that

$$
\frac{\mathrm{d}}{\mathrm{d} t} \int_{\mathbb{R}^{2}} \rho|x|^{2} \mathrm{~d} x=4 M-\frac{\chi \cos \alpha}{2 \pi} M^{2}=\frac{\chi \cos \alpha}{2 \pi} M\left(\frac{8 \pi}{\chi \cos \alpha}-M\right), \quad \forall t \in\left(0, T_{\max }\right) .
$$

Integrating (3.9) with respect to time, we arrive at the conclusion (3.5).

Proof. (Proof of Theorem 1.1.) Based on Lemma 2.1, Proposition 3.1 and Lemma 3.1, we are in a position to prove our main result Theorem 1.1.

Case 1. $\alpha \in\left(-\frac{\pi}{2}, \frac{\pi}{2}\right)$ and $M>\frac{8 \pi}{\chi \cos \alpha}$.

In view of Lemma 3.1, we can apply the moment method [4, Section 2.1] to deduce finite blow-up of $\rho$. If $T_{\max }=+\infty$, it follows from (3.5) and the fact $M>\frac{8 \pi}{\chi \cos \alpha}$ that the second moment $\int_{\mathbb{R}^{2}} \rho(x, t)|x|^{2} \mathrm{~d} x$ becomes strictly negative in finite time, which contradicts the non-negativity of $\rho$. As a consequence, the maximal time of existence is finite and it has the following upper bound:

$$
T_{\max } \leq \frac{1}{\chi(\cos \alpha) M-8 \pi}\left(\frac{2 \pi}{M}\right) \int_{\mathbb{R}^{2}} \rho_{0}|x|^{2} \mathrm{~d} x .
$$

A further application of Proposition 3.1 also yields that

$$
\lim _{t \nearrow T_{\max }} \int_{\mathbb{R}^{2}} \rho \log \rho \mathrm{d} x=+\infty .
$$

Case 2. $\alpha \in\left(-\frac{\pi}{2}, \frac{\pi}{2}\right)$ and $M<\frac{8 \pi}{\chi \cos \alpha}$.

This is the so-called subcritical mass case and the existence of global free-energy solutions can be proved by adapting the argument in [4] for $\alpha=0$. Namely, we first show that the entropy $\int_{\mathbb{R}^{2}} \rho(x, t)|\log \rho(x, t)| \mathrm{d} x$ remains bounded in time and next, we prove the propagation of $L^{p}$-norms. Taking into account that the regularization argument follows the same reasoning as in [4] (see Appendix), we just derive some a priori bounds on the solution and skip the details of the regularization procedure. 
Let us recall the two-dimensional version of the logarithmic Hardy-LittlewoodSobolev inequality (see [7]):

LEMMA 3.2. Let $f$ be a nonnegative function in $L^{1}\left(\mathbb{R}^{2}\right)$ such that $f \log f$ and $f \log (1+$ $|x|^{2}$ ) belong to $L^{1}\left(\mathbb{R}^{2}\right)$. If $\int_{\mathbb{R}^{2}} f \mathrm{~d} x=M_{*}>0$, then

$$
\int_{\mathbb{R}^{2}} f \log f \mathrm{~d} x+\frac{2}{M_{*}} \int_{\mathbb{R}^{2} \times \mathbb{R}^{2}} f(x) f(y) \log |x-y| \mathrm{d} x \mathrm{~d} y \geq-C\left(M_{*}\right),
$$

with $C\left(M_{*}\right)=M_{*}\left(1+\log \pi-\log M_{*}\right)$.

Then Lemma 3.2 combined with the monotonicity of the free energy functional $\mathcal{E}_{\alpha}$ yields

$$
\begin{aligned}
& \int_{\mathbb{R}^{2}} \rho(x, t) \log \rho(x, t) \mathrm{d} x \\
\leq & \mathcal{E}_{\alpha}\left(\rho_{0}\right)-\frac{\chi \cos \alpha}{4 \pi} \int_{\mathbb{R}^{2}} \int_{\mathbb{R}^{2}} \rho(x, t) \rho(y, t) \log |x-y| \mathrm{d} x \mathrm{~d} y \\
\leq & \mathcal{E}_{\alpha}\left(\rho_{0}\right)+\frac{\chi \cos \alpha}{8 \pi} M C(M)+\frac{\chi \cos \alpha}{8 \pi} M \int_{\mathbb{R}^{2}} \rho(x, t) \log \rho(x, t) \mathrm{d} x .
\end{aligned}
$$

Thus, we obtain the uniform estimate

$$
\int_{\mathbb{R}^{2}} \rho(x, t) \log \rho(x, t) \mathrm{d} x \leq \frac{1}{1-\frac{\chi \cos \alpha}{8 \pi} M}\left(\mathcal{E}_{\alpha}\left(\rho_{0}\right)+\frac{\chi \cos \alpha}{8 \pi} M C(M)\right),
$$

for all $t \in\left[0, T_{\max }\right)$. Applying (3.10) and [3, Lemma 2.2], we are able to control the negative part of the entropy and obtain the following estimate

$$
\int_{\mathbb{R}^{2}} \rho(x, t)|\log \rho(x, t)| \mathrm{d} x \leq \int_{\mathbb{R}^{2}} \rho(x, t)\left(\log \rho(x, t)+|x|^{2}\right) \mathrm{d} x+2 M \log (2 \pi)+\frac{2}{e} .
$$

The above inequality together with (3.5) and (3.10) implies that

$$
\rho \log \rho \in L^{\infty}\left(0, T_{\max } ; L^{1}\left(\mathbb{R}^{2}\right)\right) .
$$

In particular, the entropy functional $\int_{\mathbb{R}^{2}} \rho(x, t) \log \rho(x, t) \mathrm{d} x$ remains bounded for any finite time $t$ and thus by the criterion of extensibility stated in Proposition 3.1, we deduce that $T_{\max }=+\infty$.

Case 3. $\alpha \in\left(-\pi,-\frac{\pi}{2}\right] \cup\left[\frac{\pi}{2}, \pi\right]$.

Using the identities

$$
\operatorname{div} A \nabla c=\cos \alpha \Delta c, \quad-\Delta c=\rho,
$$

we deduce that

$$
\begin{aligned}
\frac{\mathrm{d}}{\mathrm{d} t} \int_{\mathbb{R}^{2}} \rho \log \rho \mathrm{d} x & =-4 \int_{\mathbb{R}^{2}}|\nabla \sqrt{\rho}|^{2} \mathrm{~d} x+\chi \int_{\mathbb{R}^{2}} \nabla \rho \cdot A \nabla c \mathrm{~d} x \\
& =-4 \int_{\mathbb{R}^{2}}|\nabla \sqrt{\rho}|^{2} \mathrm{~d} x-\chi \int_{\mathbb{R}^{2}} \rho \operatorname{div}(A \nabla c) \mathrm{d} x \\
& =-4 \int_{\mathbb{R}^{2}}|\nabla \sqrt{\rho}|^{2} \mathrm{~d} x+\chi \cos \alpha \int_{\mathbb{R}^{2}} \rho^{2} \mathrm{~d} x .
\end{aligned}
$$

Since for all $\alpha \in\left(-\pi,-\frac{\pi}{2}\right] \cup\left[\frac{\pi}{2}, \pi\right]$, it holds that $\cos \alpha \leq 0$, then it follows from (3.11) that the entropy $\int_{\mathbb{R}^{2}} \rho(x, t) \log \rho(x, t) \mathrm{d} x$ is decreasing in time and thus bounded from above due to the assumption (1.12) on $\rho_{0}$. Hence, we can apply the same argument described in Case 2 (cf. (3.10)) to prove the global existence of free-energy solution.

The proof of Theorem 1.1 is complete. 
Acknowledgments. The authors would like to thank the anonymous referee for his/her careful reading and helpful comments that allowed us to improve the paper. Part of the work was commenced when E. Espejo was visiting School of Mathematical Sciences and Key Laboratory of Mathematics for Nonlinear Science (Ministry of Education) at Fudan University, whose hospitality is gratefully acknowledged. H. Wu is partially supported by NNSFC grant No. 11631011 and the Shanghai Center for Mathematical Sciences.

\section{Appendix.}

4.1. Local well-posedness: proof of Proposition 3.1. To prove Proposition 3.1 , we can follow the method in $[3,4]$ for the classical parabolic-elliptic Keller-Segel model with $\alpha=0$. Roughly speaking, we first regularize the two-dimensional Newtonian potential $K$ (see (1.9)) in the formula $c=\rho * K$, solve the regularized system, derive uniform estimates with respect to the approximating parameter and then pass to the limit. In what follows, we shall briefly explain the corresponding adaptations to our problem (1.1)-(1.3) and refer for the details of this procedure to [4, Sections 2-3] and [3, Section 2].

Step 1. The regularized problem. As in [3], we choose a radial, monotone, nonincreasing smooth function such that

$$
K^{1}(x)=\left\{\begin{array}{cl}
-\frac{1}{2 \pi} \log |x| & \text { if }|x| \geq 2 \\
0 & \text { if }|x| \leq \frac{1}{2}
\end{array}\right.
$$

and

$$
\left|\nabla K^{1}(x)\right| \leq \frac{1}{2 \pi|x|}, \quad K^{1}(x) \leq-\frac{1}{2 \pi} \log |x|, \quad \forall x \in \mathbb{R}^{2}
$$

Then we define a truncated version of the potential $K(x)=-\frac{1}{2 \pi} \log |x|$ as follows

$$
K^{\varepsilon}(x):=K^{1}\left(\frac{x}{\varepsilon}\right)-\frac{1}{2 \pi} \log \varepsilon, \quad \forall \varepsilon>0,
$$

which, by its definition, fulfills

$$
\left|\nabla K^{\varepsilon}(x)\right| \leq \frac{1}{2 \pi|x|}, \quad \forall x \in \mathbb{R}^{2}, \quad \forall \varepsilon>0 .
$$

Hence, the regularized system for Equation (3.2) takes the following form:

$$
\begin{array}{ll}
\rho_{t}^{\varepsilon}=\Delta \rho^{\varepsilon}-\chi \operatorname{div}\left[\rho^{\varepsilon} A \nabla\left(\rho^{\varepsilon} * K^{\varepsilon}\right)\right], & (x, t) \in \mathbb{R}^{2} \times \mathbb{R}^{+}, \\
\rho^{\varepsilon}(x, 0)=\rho_{0}^{\varepsilon}:=\min \left\{\rho_{0}, \varepsilon^{-1}\right\}(x), & x \in \mathbb{R}^{2} .
\end{array}
$$

By construction of the initial datum $\rho_{0}^{\varepsilon}$ and assumption (1.12), it holds $\rho_{0}^{\varepsilon} \in L^{1}\left(\mathbb{R}^{2}\right) \cap$ $L^{\infty}\left(\mathbb{R}^{2}\right)$, which implies $\rho_{0}^{\varepsilon} \in L^{2}\left(\mathbb{R}^{2}\right)$. Next, keeping in mind that $A$ is an orthogonal matrix (see (1.4)), then taking the standard Euclidean $\mathbb{R}^{2}$-norm we see that

$$
\left|A \nabla\left(\rho^{\varepsilon} * K^{\varepsilon}\right)\right|=\left|\nabla\left(\rho^{\varepsilon} * K^{\varepsilon}\right)\right|=\left|\rho^{\varepsilon} * \nabla K^{\varepsilon}\right| .
$$

Thus, by Young's inequality for convolution, we get

$$
\left\|A \nabla\left(\rho^{\varepsilon} * K^{\varepsilon}\right)\right\|_{L^{\infty}\left(\mathbb{R}^{2}\right)} \leq\left\|\rho^{\varepsilon}\right\|_{L^{1}\left(\mathbb{R}^{2}\right)}\left\|\nabla K^{\varepsilon}\right\|_{L^{\infty}\left(\mathbb{R}^{2}\right)} .
$$


Therefore, we can mimic the same proof for the case of chemotactic response without rotation in [4, Section 2.5], which is based on Schauder's fixed-point theorem and the Lions-Aubin compactness method, to conclude that, for any $\varepsilon>0, \alpha \in(-\pi, \pi]$ and $T>0$, the regularized problem (4.2)-(4.3) admits a unique global solution

$$
\rho^{\varepsilon} \in C\left([0, T] ; L^{2}\left(\mathbb{R}^{2}\right)\right) \cap L^{2}\left(0, T ; H^{1}\left(\mathbb{R}^{2}\right)\right),
$$

provided that $\rho_{0}$ satisfies (1.12). Moreover, the approximating solution $\rho^{\varepsilon}$ is nonnegative and its mass is conserved:

$$
0 \leq \int_{\mathbb{R}^{2}} \rho^{\varepsilon}(x, t) \mathrm{d} x=\int_{\mathbb{R}^{2}} \rho^{\varepsilon}(x, 0) \mathrm{d} x \leq \int_{\mathbb{R}^{2}} \rho_{0} \mathrm{~d} x=M, \quad \forall t \in[0, T] .
$$

Step 2. Uniform estimates in $\varepsilon$. We only explain some necessary modifications that have to be done when taking into account the role of matrix $A$.

First, for the global solution $\rho^{\varepsilon}$ to the regularized problem (4.2)-(4.3), we notice that the associated free energy functional given by

$$
\mathcal{E}_{\alpha}^{\varepsilon}\left(\rho^{\varepsilon}\right):=\int_{\mathbb{R}^{2}} \rho^{\varepsilon} \log \rho^{\varepsilon} \mathrm{d} x-\frac{\chi \cos \alpha}{2} \int_{\mathbb{R}^{2}} \rho^{\varepsilon}\left(\rho^{\varepsilon} * K^{\varepsilon}\right) \mathrm{d} x,
$$

satisfies the dissipative property

$$
\frac{\mathrm{d}}{\mathrm{d} t} \mathcal{E}_{\alpha}^{\varepsilon}\left(\rho^{\varepsilon}\right)=-\int_{\mathbb{R}^{2}} \rho^{\varepsilon}\left|\nabla \log \rho^{\varepsilon}-\chi \cos \alpha \nabla\left(\rho^{\varepsilon} * K^{\varepsilon}\right)\right|^{2} \mathrm{~d} x \leq 0,
$$

whose justification follows the same reasoning applied for finding (2.1).

Second, we derive an estimate for the second moment of $\rho^{\varepsilon}$. Similar to (3.6), we have

$$
\begin{aligned}
\frac{\mathrm{d}}{\mathrm{d} t} \int_{\mathbb{R}^{2}} \rho^{\varepsilon}|x|^{2} \mathrm{~d} x & =4 \int_{\mathbb{R}^{2}} \rho^{\varepsilon} \mathrm{d} x+2 \chi \int_{\mathbb{R}^{2}} x \cdot\left[\rho^{\varepsilon}(x, t) \int_{\mathbb{R}^{2}} \rho^{\varepsilon}(y, t) A \nabla K^{\varepsilon}(x-y) \mathrm{d} y\right] \mathrm{d} x \\
& =4 \int_{\mathbb{R}^{2}} \rho_{0}^{\varepsilon} \mathrm{d} x+\chi \int_{\mathbb{R}^{2}} \int_{\mathbb{R}^{2}} \rho^{\varepsilon}(x, t) \rho^{\varepsilon}(y, t)(x-y) \cdot\left[A \nabla K^{\varepsilon}(x-y)\right] \mathrm{d} x \mathrm{~d} y \\
& \leq 4 M+\chi \int_{\mathbb{R}^{2}} \int_{\mathbb{R}^{2}} \rho^{\varepsilon}(x, t) \rho^{\varepsilon}(y, t)(x-y) \cdot\left[A \nabla K^{\varepsilon}(x-y)\right] \mathrm{d} x \mathrm{~d} y .
\end{aligned}
$$

Besides, it follows from (1.4) and (4.1) that

$$
(x-y) \cdot A \nabla K^{\varepsilon}(x-y) \leq|x-y|\left|\nabla K^{\varepsilon}(x-y)\right| \leq \frac{1}{2 \pi} .
$$

From the above two inequalities and (4.4), we infer that

$$
\begin{aligned}
\frac{\mathrm{d}}{\mathrm{d} t} \int_{\mathbb{R}^{2}} \rho^{\varepsilon}|x|^{2} \mathrm{~d} x & \leq 4 M+\frac{\chi}{2 \pi} \int_{\mathbb{R}^{2}} \int_{\mathbb{R}^{2}} \rho^{\varepsilon}(x, t) \rho^{\varepsilon}(y, t) \mathrm{d} x \mathrm{~d} y \\
& \leq 4 M+\frac{\chi}{2 \pi} M^{2} .
\end{aligned}
$$

Third, using again the identity $\operatorname{div}(A \nabla f)=\cos \alpha \Delta f$, we obtain the following estimate on the entropy functional $\int_{\mathbb{R}^{2}} \rho^{\varepsilon} \log \rho^{\varepsilon} \mathrm{d} x$ :

$$
\frac{\mathrm{d}}{\mathrm{d} t} \int_{\mathbb{R}^{2}} \rho^{\varepsilon} \log \rho^{\varepsilon} \mathrm{d} x=-4 \int_{\mathbb{R}^{2}}\left|\nabla \sqrt{\rho^{\varepsilon}}\right|^{2} \mathrm{~d} x+\chi \int_{\mathbb{R}^{2}} \nabla \rho^{\varepsilon} \cdot\left[A \nabla\left(\rho^{\varepsilon} * K^{\varepsilon}\right)\right] \mathrm{d} x
$$




$$
\begin{aligned}
& =-4 \int_{\mathbb{R}^{2}}\left|\nabla \sqrt{\rho^{\varepsilon}}\right|^{2} \mathrm{~d} x-\chi \int_{\mathbb{R}^{2}} \rho^{\varepsilon} \operatorname{div}\left[A \nabla\left(\rho^{\varepsilon} * K^{\varepsilon}\right)\right] \mathrm{d} x \\
& =-4 \int_{\mathbb{R}^{2}}\left|\nabla \sqrt{\rho^{\varepsilon}}\right|^{2} \mathrm{~d} x-\chi \cos \alpha \int_{\mathbb{R}^{2}} \rho^{\varepsilon} \Delta\left(\rho^{\varepsilon} * K^{\varepsilon}\right) \mathrm{d} x .
\end{aligned}
$$

In particular, the term $\int_{\mathbb{R}^{2}} \rho^{\varepsilon} \Delta\left(K^{\varepsilon} * \rho^{\varepsilon}\right) \mathrm{d} x$ on the right-hand side of the above identity can be handled exactly as in the proof of [3, Lemma 2.3].

Step 3. Proof of Proposition 3.1.

Proof. Keeping the above modifications in mind, we are able to repeat the arguments in [3, Sections 2.2-2.3] (with some further details in [4, Sections 2-3]) to conclude the existence of free-energy solutions to problem (1.1)-(1.3) on the maximal time interval $\left[0, T_{\max }\right)$. Besides, we note that the uniqueness of free-energy solutions follows from the same techniques as in [11, Theorem 1.3]. The details are omitted here.

The proof of Proposition 3.1 is complete.

4.2. Model derivation: an energetic variational approach. In the last part of this paper, we describe a (formal) derivation of system (1.1)-(1.2) by using the so-called energetic variational approach, which combines the least action principle and Onsager's principle of maximum energy dissipation in continuum mechanics (see for instance, $[14,18,27]$ and the references therein). Through the derivation, it is easy to see that system (1.1)-(1.2) naturally fulfills three important physical constraints such as mass conservation, energy dissipation and force balance.

(A) Mass conservation. We simply impose the generic kinematic relation, i.e., the conservation of mass, in the Eulerian coordinate such that

$$
\rho_{t}+\operatorname{div}(\rho u)=0, \quad(x, t) \in \mathbb{R}^{2} \times(0, T),
$$

where $u: \mathbb{R}^{2} \times(0, T) \rightarrow \mathbb{R}^{2}$ stands for the effective velocity, for instance, due to possible mass diffusion and drift effects etc.

(B) Energy dissipation. Next, we impose the following abstract dissipative energy law (cf. $[8,18])$

$$
\frac{\mathrm{d}}{\mathrm{d} t} \mathcal{E}=-\mathcal{D}, \quad \forall t \in(0, T)
$$

with the free energy and the energy dissipation given by

$$
\mathcal{E}(\rho):=\int_{\mathbb{R}^{2}} \omega(\rho) \mathrm{d} x, \quad \mathcal{D}(\rho, u):=\int_{\mathbb{R}^{2}} \eta(\rho)|u-v(\rho)|^{2} \mathrm{~d} x .
$$

Specific form of the energy density function $\omega$, the extra velocity field $v$ (due to possible correction induced by $\rho$ ) and the (nonnegative) coefficient $\eta$ will be chosen below.

(C) Force balance. Let us now introduce the flow map $x(X, t): \mathbb{R}^{2} \rightarrow \mathbb{R}^{2}, t \geq 0$, which is a solution to the ordinary differential equation $x_{t}(X, t)=u(x(X, t), t)$ subject to the initial condition $x(X, 0)=X$. Here, $X$ is the Lagrangian coordinate system (the reference configuration) and $x$ stands for the Eulerian coordinate system (the deformed configuration). The deformation gradient of the flow map $x(X, t)$ is given by

$$
\mathrm{F}(X, t):=\frac{\partial x(X, t)}{\partial X} .
$$

Then from (4.5) and a change of coordinates we see that

$$
\rho(x(X, t), t)=\frac{\rho_{0}(X)}{\operatorname{detF}}, \quad \forall t \geq 0 .
$$


The least action principle states that the trajectories of particles from the position $x(X, 0)$ at time $t=0$ to $x(X, T)$ at a given time $T$ in a Hamiltonian system are those that minimize the action functional:

$$
\mathcal{A}(x(X, t)):=-\int_{0}^{T} \mathcal{E}(\rho) \mathrm{d} t=-\int_{0}^{T} \int_{\mathbb{R}^{2}} \omega\left(\frac{\rho_{0}(X)}{\operatorname{detF}}\right) \operatorname{detF} \mathrm{d} X \mathrm{~d} t .
$$

Taking variation of the action $\mathcal{A}$ with respect to the flow map $x$ leads to the conservative force (cf. [27, Section 2.2.1]) such that $\delta_{x} \mathcal{A}:=F_{\text {conv }} \cdot \delta x$. Here, since the macroscopic kinetic energy is assumed to be neglected in our system, the associated inertial force simply vanishes. Then by a direct computation of $\delta_{x} \mathcal{A}$ (see e.g., [14,18]), we obtain the generalized conservative force (written as a strong form in the Eulerian coordinate):

$$
F_{\text {conv }}=-\rho \nabla\left(\delta_{\rho} \mathcal{E}\right)
$$

On the other hand, we can also include dissipative forces in the system, through Onsager's maximum dissipation principle. This is done by taking variation of the Rayleigh dissipation functional $\mathcal{R}:=\frac{1}{2} \mathcal{D}$ with respect to the rate function, i.e., the velocity $u$ such that $\delta_{u} \mathcal{R}:=-F_{\text {diss }} \cdot \delta u$ (cf. [27, Section 2.2.1]). Together with (4.6), we get

$$
F_{\text {diss }}=-\eta(\rho)[u-v(\rho)]
$$

Hence, from the classical Newton's force balance law $F_{\text {conv }}+F_{\text {diss }}=0$, we deduce that

$$
\eta(\rho) u=-\rho \nabla\left(\delta_{\rho} \mathcal{E}\right)+\eta(\rho) v(\rho) .
$$

In particular, the relation (4.7) enables us to determine an exact form of the velocity $u$, which together with (4.5) yields a closed partial differential equation for $\rho$.

Derivation of system (1.1)-(1.2). In the above general framework, we now choose

$$
\omega(\rho)=\rho \log \rho-\frac{\chi \cos \alpha}{2} \rho(\rho * K), \quad v(\rho)=\chi(\sin \alpha) R \nabla(\rho * K), \quad \eta(\rho)=\rho,
$$

with $\chi>0, \alpha \in(-\pi, \pi]$. The kernel function $K$ representing the nonlocal interaction is given by (1.9) and the matrix $R$ is taken as in (2.5), inducing possible velocity correction along the direction orthogonal to $\nabla(\rho * K)$. By a direct calculation, we get

$$
\delta_{\rho} \mathcal{E}=1+\log \rho-\chi \cos \alpha(\rho * K) .
$$

Then recalling the definition of the rotational matrix $A$ (i.e., (1.4)) and the decomposition (2.6), we infer from (4.7)-(4.9) that

$$
\begin{aligned}
\rho u & =-\nabla \rho+\chi(\cos \alpha) \rho I \nabla(\rho * K)+\chi(\sin \alpha) \rho R \nabla(\rho * K) \\
& =-\nabla \rho+\chi \rho A \nabla(\rho * K) .
\end{aligned}
$$

Inserting the above relation back into (4.5), we arrive at Equation (3.2), which is equivalent to our system (1.1)-(1.2) in view of (3.1).

\section{REFERENCES}

[1] N. Bellomo, A. Bellouquid, Y.-S. Tao, and M. Winkler, Toward a mathematical theory of KellerSegel models of pattern formation in biological tissues, Math. Models Meth. Appl. Sci., 25(9):1663-1763, 2015. 1 
[2] P. Biler and J. Dolbeault, Long time behavior of solutions to Nernst-Planck and Debye-Hückel drift-diffusion systems, Ann. Henri Poincaré, 1:461-472, 2000. 1

[3] A. Blanchet, J.-A. Carrillo, and N. Masmoudi, Infinite time aggregation for the critical PatlakKeller-Segel model in $\mathbb{R}^{2}$, Commun. Pure Appl. Math., 61:1449-1481, 2008. 1, 1, 1, 3, 3, 3.1, $3,3,4.1,4.1$

[4] A. Blanchet, J. Dolbeault, and B. Perthame, Two-dimensional Keller-Segel model: optimal critical mass and qualitative properties of the solutions, Electron. J. Diff. Eqs., 44:32, 2006. 1, 1, 1, 3, 3, 3.1, 3, 3, 4.1, 4.1, 4.1

[5] X.-R. Cao and S. Ishida, Global-in-time bounded weak solutions to a degenerate quasilinear KellerSegel system with rotation, Nonlinearity, 27:1899-1913, 2014. 1

[6] X.-R. Cao and J. Lankeit, Global classical small-data solutions for a three-dimensional chemotaxis Navier-Stokes system involving matrix-valued sensitivities, Calc. Var. Part. Diff. Eqs., 55:107, 2016. 1

[7] E. Carlen and M. Loss, Competing symmetries, the logarithmic HLS inequality and Onofri's inequality on $S^{n}$, Geom. Funct. Anal., 2:90-104, 1992. 3

[8] C. Deng and C. Liu, Largest well-posed spaces for the general diffusion system with nonlocal interactions, J. Funct. Anal., 272(10):4030-4062, 2017. 1, 1, 4.2

[9] W.-R. DiLuzio, L. Turner, M. Mayer, P. Garstecki, D.-B. Weibel, H.-C. Berg, and G.-M. Whitesides, Escherichia coli swim on the right-hand side, Nature, 435:1271-1274, 2005. 1

[10] Y. Dong and Z.-Y. Xiang, Global large-data generalized solutions in a chemotactic movement with rotational flux caused by two stimuli, Nonlinear Anal. Real World Appl., 41:549-569, 2018. 1

[11] G.-E. Fernández and S. Mischler, Uniqueness and long time asymptotic for the Keller-Segel equation: the parabolic-elliptic case, Arch. Ration. Mech. Anal., 220:1159-1194, 2016. 1, 1, 3, 3, 3.1, 4.1

[12] P.-D. Frymier, R.-M. Ford, H.-C. Berg, and P.-T. Cummings, Three-dimensional tracking of motile bacteria near a solid planar surface, Proc. Natl. Acad. Sci. USA, 92:6195-6199, 1995. 1

[13] M.-H. Giga, Y. Giga, and J. Saal, Nonlinear Partial Differential Equations: Asymptotic Behavior of Solutions and Self-similar Solutions, Birkhäuser, Basel, 2010. 1, 1

[14] M.-H. Giga, A. Kirshtein, and C. Liu, Variational modeling and complex fluids, in Y. Giga and A. Novotný (eds.), Handbook of Mathematical Analysis in Mechanics of Viscous Fluids, Springer, 73-113, 2018. 1, 4.2, 4.2

[15] T. Hillen and K.-J. Painter, A user's guide to PDE models for chemotaxis, J. Math. Biol., 58:183217, 2009. 1

[16] D. Horstmann, From 1970 until present: the Keller-Segel model in chemotaxis and its consequences I, Jahresber. Dtsch. Math. Ver., 105:103-165, 2003. 1

[17] D. Horstmann, From 1970 until present: the Keller-Segel model in chemotaxis and its consequences II, Jahresber. Dtsch. Math. Ver., 106:51-69, 2004. 1

[18] Y. Hyon, D.-Y. Kwak, and C. Liu, Energetic variational approach in complex fluids: maximum dissipation principle, Discrete Contin. Dyn. Syst. Ser. A, 26:1291-1304, 2010. 1, 4.2, 4.2, 4.2

[19] W. Jäger and S. Luckhaus, On explosions of solutions to a system of partial differential equations modelling chemotaxis, Trans. Amer. Math. Soc., 329(2):819-824, 1992. 1

[20] E.-F. Keller and L.-A. Segel, Initiation of slide mold aggregation viewed as an instability, J. Theor. Biol., 26:399-415, 1970. 1

[21] T. Li, A. Suen, M. Winkler, and C. Xue, Global small-data solutions of a two-dimensional chemotaxis system with rotational flux terms, Math. Models Meth. Appl. Sci., 25(4):721-746, 2015. 1

[22] P.-A. Markowich, C.-A. Ringhofer, and C. Schmeiser, Semiconductor Equations, Springer, New York, 1990. 1

[23] T. Nagai, Global existence and decay estimates of solutions to a parabolic-elliptic system of driftdiffusion type in $\mathbb{R}^{2}$, Diff. Int. Eqs., 24(1-2):29-68, 2011. 1

[24] T. Nagai and T. Ogawa, Global existence of solutions to a parabolic-elliptic system of driftdiffusion type in $\mathbb{R}^{2}$, Funkcial. Ekvac., 59(1):67-112, 2016. 1

[25] T. Nagai and T. Yamada, Boundedness of solutions to a parabolic-elliptic Keller-Segel equation in $\mathbb{R}^{2}$ with critical mass, Adv. Nonlinear Stud., 18(2):337-360, 2018. 1

[26] C.-S. Patlak, Random walk with persistence and external bias, Bull. Math. Biophys., 15:311-338, 1953. 1

[27] A.-M. Sonnet and E.-G. Virga, Dissipative Ordered Fluids Theories for Liquid Crystals, SpringerVerlag, New York, 2012. 4.2, 4.2

[28] Y.-L. Wang, Global bounded weak solutions to a degenerate quasilinear chemotaxis system with rotation, Math. Meth. Appl. Sci., 39(5):1159-1175, 2016. 1

[29] Y.-L. Wang and X.-R. Cao, Global classical solutions of a 3D chemotaxis-Stokes system with rotation, Discrete Contin. Dyn. Syst. Ser. B, 20(9):3235-3254, 2015. 1 
[30] Y.-L. Wang and Z.-Y. Xiang, Global existence and boundedness in a Keller-Segel-Stokes system involving a tensor-valued sensitivity with saturation, J. Diff. Eqs., 259(12):7578-7609, 2015. 1

[31] Y.-L. Wang and Z.-Y. Xiang, Global existence and boundedness in a Keller-Segel-Stokes system involving a tensor-valued sensitivity with saturation: the 3D case, J. Diff. Eqs., 261(9):49444973, 2016. 1

[32] M. Winkler, Large-data global generalized solutions in a chemotaxis system with tensor-valued sensitivities, SIAM J. Math. Anal., 47(4):3092-3115, 2015. 1

[33] M. Winkler, Global mass-preserving solutions in a two-dimensional chemotaxis-Stokes system with rotational flux components, J. Evol. Eqs., 18(3):1267-1289, 2018. 1

[34] C. Xue and H.-G. Othmer, Multiscale models of taxis-driven patterning in bacterial populations, SIAM J. Appl. Math., 70:133-167, 2009. 1

[35] Q.-S. Zhang, Boundedness in chemotaxis systems with rotational flux terms, Math. Nachr., 289:2323-2334, 2016. 1 